\title{
Kinetics of photoinduced matter transport driven by intensity and polarization in thin films containing azobenzene
}

\author{
F. Fabbri, ${ }^{1}$ Y. Lassailly, ${ }^{1}$ S. Monaco, ${ }^{2}$ K. Lahlil,${ }^{1}$ J. P. Boilot, ${ }^{1}$ and J. Peretti ${ }^{1, *}$ \\ ${ }^{1}$ Physique de la Matière Condensée, CNRS-Ecole Polytechnique, 91128 Palaiseau Cédex, France \\ ${ }^{2}$ Dipartimento di Informatica e Sistemistica, Università La Sapienza, Via Ariosto 25, 00185 Rome, Italy
}

(Received 7 June 2012; published 24 September 2012)

\begin{abstract}
We investigate the kinetics of photoinduced deformation phenomena in azobenzene-containing thin solid films. We show that a light intensity pattern and a light polarization pattern produce two distinct material transport processes whose direction and kinetics can be independently controlled. The kinetics of the intensity-driven deformation scales with the incoming light power while the kinetics of the polarization-driven mass transport scales with the amplitude of the electromagnetic field pattern. We conclude that these two processes are fully independent one from the other and originate from two different microscopic mechanisms.
\end{abstract}

DOI: 10.1103/PhysRevB.86.115440

\section{INTRODUCTION}

Amorphous materials containing azobenzene derivatives reveal uncommon photomechanical properties under illumination in the chromophore absorption band. In particular, the holographic projection of an optical pattern on a thin film of an azobenzene-containing material produces a surface relief, which may reach amplitudes almost as large as the film thickness. ${ }^{1,2}$ This deformation results from an in-plane photoinduced mass transport across the optical pattern. The microscopic origin of this phenomenon is still not elucidated. It is usually stated that the material transport is related to repeated photoisomerization cycles of the photochromes, although the actual role of the photoisomerization process is still to be debated. Similar behaviors are observed in other types of materials, which also exhibit a strong photoinduced change in the conformation at the molecular level..$^{3-5}$ The photomechanical response of azobenzene-containing materials is generally studied by measuring the formation of a surface relief grating (SRG) under projection of a two-beam interference pattern. Most studies emphasize the role of the light polarization and report that efficient material transport requires a light electric field component parallel to the grating vector. ${ }^{6-8}$ Correlatively, the material photomechanical response to a pure intensity pattern obtained with $s$-polarized beams (i.e., with the electric field perpendicular to the grating vector) is claimed to be very weak and is not considered. These conclusions rely on indirect measurements of the photoinduced deformation kinetics obtained by monitoring the diffraction efficiency of the SRG as a function of exposure time. The moderate diffraction efficiencies usually observed under excitation with $s$-polarized pure intensity interference patterns are often attributed to the spatial modulation of the material refractive index. ${ }^{7}$ However, by using in situ near-field techniques, which allow direct observation of the actual surface relief formation under illumination, ${ }^{9-11}$ we have recently evidenced that illumination with an $s$-polarized pure intensity interference pattern may in fact produce in-plane directional mass transport. ${ }^{12}$ The efficiency of this process is found to strongly depend on the nature of the host matrix in contrast with the mass transport produced by illumination with a polarization pattern. We have thus proposed the existence of two distinct directional mass transport mechanisms, one triggered by the light intensity pattern and the other by the light polarization pattern. Nevertheless, the two-beam interference configuration does not allow us to fully disentangle the respective contributions of the light polarization pattern and of the light intensity pattern to the photodeformation kinetics. ${ }^{13}$

In the present paper, we study the photomechanical response of azobenzene-containing materials in the so-called assisting-beam illumination configuration. ${ }^{10,14} \mathrm{~A}$ pure intensity interference pattern produced by two $s$-polarized coherent beams is projected onto the photochromic thin film. A third beam of different wavelength and of orthogonal polarization is superimposed at normal incidence onto the interference pattern without interfering with it. This assisting beam confers a uniform illumination, which alone does not produce the formation of any surface relief (although spontaneous patterning under single-beam illumination has been sometimes reported). ${ }^{15}$ The combination of the assisting beam with the interference pattern introduces a polarization pattern with an electric field component oriented along the grating vector of the intensity pattern. This is the key feature that allows us to fully disentangle the polarization and intensity effects on the photoinduced mass transport. Indeed, we show that the full kinetics of the deformations produced by the intensity pattern and by the polarization pattern can be separately deduced from the measurements performed with the assisting beam on and off. It is found that the two deformation processes are totally distinct one from the other. First, in two different materials, the polarization-driven process is found to be very similar while very different photomechanical responses to the intensity pattern are observed. Second, the efficiency of each process exhibits a specific dependence on the light intensity. Third, the transport directions of the two processes are independent one from the other. In the assisting-beam configuration, intensity- and polarization-driven processes produce mass transport in opposite directions with different kinetics. Their competition results in two subsequent deformation regimes, each mechanism overcoming the other at different exposure time scales, while the optical pattern is kept unchanged. A nonmonotonous deformation kinetics is thus observed with a reversal in the mass transport direction. Such a behavior cannot be explained in the framework of a single-mechanism scheme. We thus finally conclude that the two processes originate from two different microscopic mechanisms. On the basis of our 
results, we discuss the relevance of different models proposed in the literature to describe each of the processes that we experimentally observe.

\section{EXPERIMENT}

The experiments are performed on two different materials: a mineral matrix of sol-gel silica and a PMMA-based organic polymer. Both contain the same azobenzene derivative, Disperse Red 1 (DR1), grafted to the backbone. These two materials, hereafter respectively denoted Si-DR1 [Fig. 1(e)] and PMMA-DR1 [Fig. 1(i)], are spin coated on a glass substrate. In both cases, thin films have a thickness of $200 \mathrm{~nm}$, a DR1 volume concentration of $10^{21}$ molecule $/ \mathrm{cm}^{3}$, and exhibit almost identical optical absorption spectra. Details on the material synthesis are given elsewhere. ${ }^{12,16,17}$

The intensity interference pattern results from the recombination of two $s$-polarized beams [Fig. 1(a)] obtained from a split laser beam at a wavelength $\lambda_{I}=473 \mathrm{~nm}$, close to the maximum of the dye visible absorption band. The incidence angle $\theta=16.5^{\circ}$ yields a spatial interference period $\Lambda=\lambda_{I} / 2 \sin \theta=830 \mathrm{~nm}$ along the $x$ axis. Each beam power density is $1 \mathrm{~mW} / \mathrm{mm}^{2}$. The two interfering beams are linearly polarized along the $y$ axis (the so-called $s$ polarization). The interference pattern profile is given by $I_{I}^{y}=4 I_{0} \cos ^{2}(\pi x / \Lambda)$, $I_{0}$ being the intensity when illuminating with only one of the two interfering beams. It corresponds to a pure-intensity pattern exhibiting a contrast equal to 1 between bright and dark fringes.

The assisting beam illuminates the sample at normal incidence [Fig. 1(d)]. Its wavelength $\lambda_{A}=532 \mathrm{~nm}$ is different from the one of the interfering beams but still in the absorption band of the DR1 molecule. ${ }^{18}$ Its power density is $I_{A}^{x}=$ $2 \mathrm{~mW} / \mathrm{mm}^{2}$, that is equivalent to the average power density of the interference pattern. Its polarization (that we denote in the following as $p$ polarization since it is parallel to the incidence plane that contains the three beams) is oriented along the $x$ axis and is orthogonal to the one of the interfering beams.
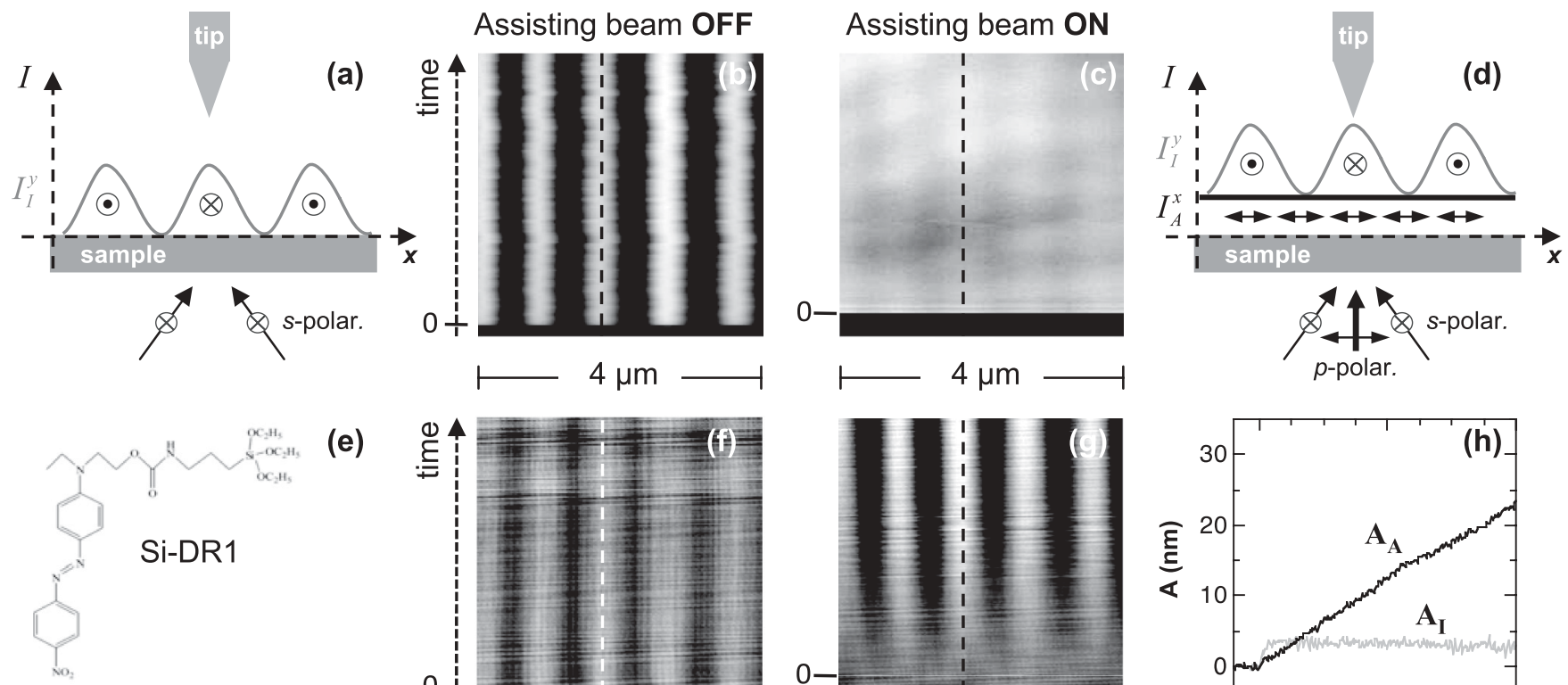

(e)
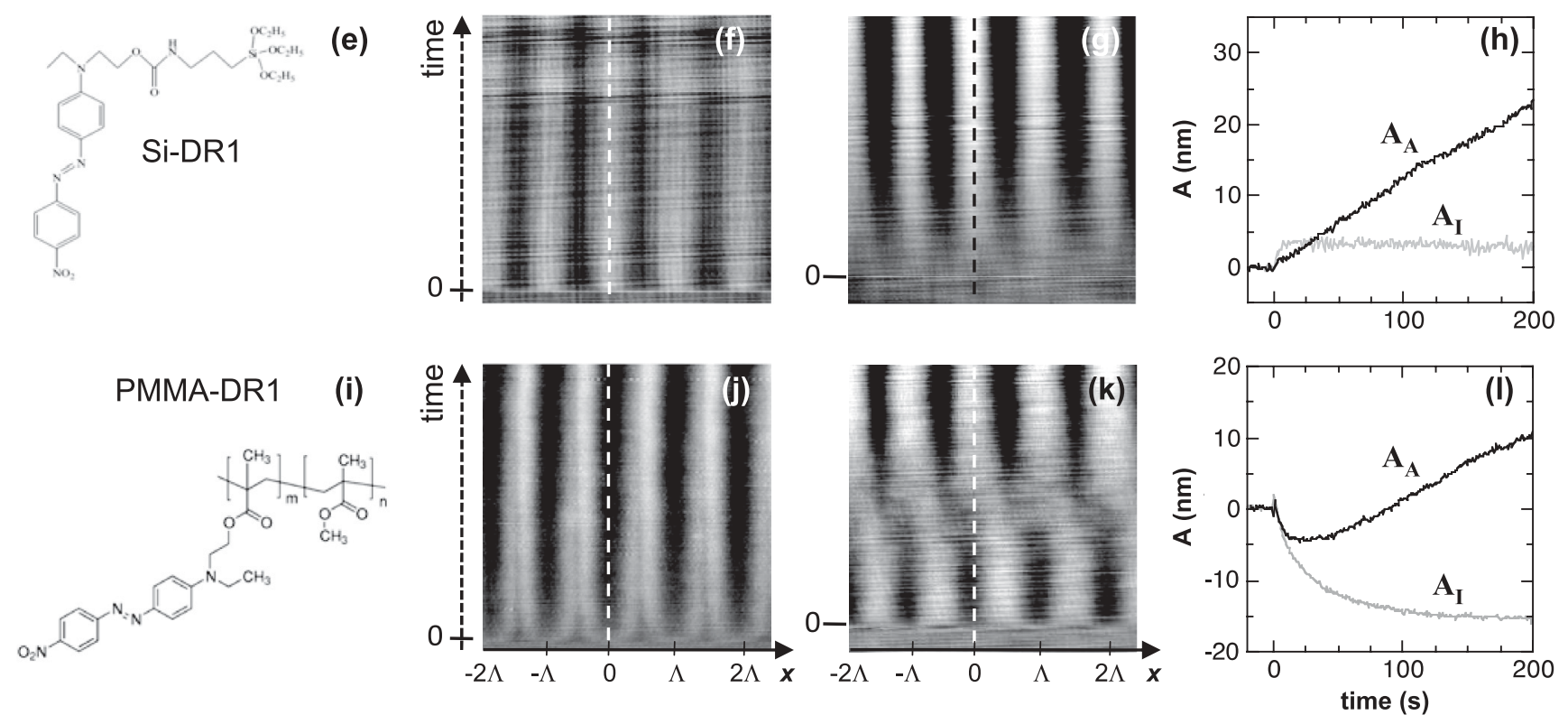

FIG. 1. (a) and (d) Schematics of the experimental setup showing the optical pattern (intensity and field orientation) at the sample surface when the assisting beam is off and on, respectively. (b) and (c) 4- $\mu$ m-wide images of the optical intensity collected by the fiber tip near the sample surface when the assisting beam is off and on, respectively. The dashed line at $x=0$ indicates the spatial reference used to determine the SRG position with respect to the $s$-polarized interference pattern. (f) and (g) Topographical images recorded during the first $200 \mathrm{~s}$ of illumination for Si-DR1, when the assisting beam is off and on, respectively. The variation of the topography signal along the image vertical axis directly yields the relief pattern evolution as a function of exposure time. (j) and (k) Same as (f) and (g) for the PMMA-DR1 sample. (h) Time evolution of the SRG amplitude $A$ in Si-DR1, extracted from (f), when the assisting beam is off (gray line) and from (g), when the assisting beam is on (dark line). (l) Same as (h) for PMMA-DR1. (e) and (i) Molecular structures of Si-DR1 and PMMA-DR1 respectively. 
The superimposition of the assisting beam onto the interfering beams produces a light pattern where areas illuminated with both field components, respectively $E_{A} \cdot \mathbf{x}$ and $E_{I} \cdot \mathbf{y}$, alternate with areas illuminated with only the $E_{A} \cdot \mathbf{x}$ component. It thus yields the combination of a polarization pattern with the intensity interference pattern. Note that in this configuration the amplitude of the $x$ component of the electric field does not exhibit any variation along the direction of the grating vector.

Upon illumination, the material photomechanical response will give rise to the formation of a SRG. In our experiment, the sample is illuminated from the back through the glass substrate [Figs. 1(a) and 1(d)], which leaves free access to the sample surface. We use in situ near-field microscopy techniques, ${ }^{19}$ which allow simultaneous detection of both the surface topography and the optical field, to track in real time the photoinduced deformation process. The measurement of the surface topography as a function of exposure time yields the kinetics and efficiency of the deformation process while the detection of the spatial-phase shift between the topography and optical patterns directly yields the mass transport direction. ${ }^{9,10,12}$

\section{RESULTS}

We start with the assisting beam off [Fig. 1(a)]. In this configuration the $s$-polarized pure intensity interference pattern is projected onto the sample. Figure 1(b) shows the experimental near-field optical image. The measured intensity contrast is $\simeq 0.98$, very close to the expected value of 1 . The surface reliefs that form in these illumination conditions on the Si-DR1 and PMMA-DR1 samples are shown respectively in Figs. 1(f) and 1(j). The kinetics of the SRG formation (i.e., the variation of the $S R G$ amplitude $A$ as a function of exposure time) is deduced from the topography images. By convention, we consider as the absolute coordinate reference along the $x$ axis the position of the light intensity maximum (i.e., bright fringes) of the $s$-polarized interference pattern. This is indicated (modulo $\Lambda$ ) by the dashed line at $x=0$ in the optical and topographical images. The SRG amplitude $A$ is defined, at exposure time $t$, as the difference between the surface profile height $h$ at the position $x=0$ and at the position $x=\Lambda / 2$ : $A(t)=h(x=0, t)-h(x=\Lambda / 2, t)$. Note that a deformation such that tops and valleys coincide respectively with bright and dark fringes of the $s$-polarized interference pattern yields positive $A$ values, while a deformation spatially shifted by $\Lambda / 2$ with respect to the $s$-polarized interference pattern yields negative $A$ values. The variation of $A(t)$ over the image acquisition time is plotted in Figs. 1(h) and 1(l) (gray lines) for Si-DR1 and PMMA-DR1 respectively.

In both materials, as the illumination is turned on, a SRG of positive amplitude rapidly forms. After only a few seconds, it reaches a saturated amplitude of about 3-4 nm (i.e., about $2 \%$ of the film thickness). This is due to the matrix photoexpansion that takes place in the illuminated areas giving rise to a SRG in coincidence with the interference pattern. ${ }^{9}$ Subsequently to the photoexpansion, the Si-DR1 material does not show any further photodeformation, while the PMMA-DR1 sample exhibits the formation of another SRG pattern shifted by $\Lambda / 2$ with respect to the initial photoexpansion pattern, as indicated by the change in the sign of $A$. This phenomenon is attributed to lateral material transport. It is directed from the bright fringes towards the dark fringes of the interference pattern and yields a deformation amplitude much larger than the photoexpansion. This result leads to two inferences. First, it shows that mass transport can be photoinduced even with $s$-polarized interfering beams (i.e., when the electric field is orthogonal to the grating vector $\mathbf{u}_{\mathrm{G}}$ ) while it is often stated that an electric field component along the grating vector is required. Second, the material transport is here induced by a pure intensity pattern, which indicates the existence of an intensity-driven mechanism whose efficiency depends on the nature of the matrix.

When projecting simultaneously the $p$-polarized assisting beam and the $s$-polarized intensity pattern [Fig. 1(d)] a light polarization pattern is achieved. The corresponding measured optical image is shown in Fig. 1(c). As compared with Fig. 1(b), the intensity contrast is lowered to about $1 / 3$ because of the intensity offset of the assisting beam. Figures $1(\mathrm{~g})$ and $1(\mathrm{k})$ show the evolution of the surface topography versus exposure time obtained respectively on the Si-DR1 and PMMA-DR1 films. The corresponding photodeformation kinetics are plotted in Figs. 1(h) and 1(1) (dark lines) together with the ones obtained in the previous experiments performed with the assisting beam off (gray lines). In both materials, as soon as the illumination is turned on, photoexpansion occurs. Since the intensity pattern contrast is low, photoexpansion produces a transient SRG whose positive amplitude does not exceed $2 \mathrm{~nm}$ before vanishing. Subsequently, in Si-DR1, a SRG goes on increasing with a positive amplitude. The positive sign of $A$ indicates that the relief pattern coincides with the $s$-polarized interference pattern. This phenomenon is attributed to a directional mass migration mechanism fully governed by the polarization pattern, which drives material from areas illuminated by a light field parallel to the grating vector $\mathbf{u}_{\mathrm{G}}$ (here the $E_{A} \cdot \mathbf{x}$ field of the assisting beam in the dark fringes of the interference pattern) toward areas illuminated by a light field having a component perpendicular to $\mathbf{u}_{\mathrm{G}}$ (here the interference field $E_{I} \cdot \mathbf{y}$ ). This is in agreement with previously reported data. ${ }^{10,20}$ It is important to note that the material transport here takes place from the darkest areas towards the brightest areas, which is the opposite direction of the mass transport produced by the pure intensity pattern in PMMA-DR1 [Fig. 1(j)]. It is thus clear that in Si-DR1 material, the mass transport direction and efficiency are fully determined by the polarization pattern. In PMMADR1 [Fig. 1(k)], following the photoexpansion, the SRG amplitude changes sign to become negative. Then, it vanishes and again changes sign to finally form a SRG of positive amplitude in coincidence with the $s$-polarized interference pattern. The negative deformation regime results from the intensity-driven mass migration observed in the nonassisted experiment [Fig. 1(d)]. The subsequent positive deformation is induced by the polarization-driven material transport, similarly to what we observe in the Si-DR1 sample.

In PMMA-DR1, photoinduced mass transport can thus be produced by both polarization and intensity patterns. In the illumination configuration used here, these two effects compete against each other, which induces a reversal of the material transport direction while the illumination 


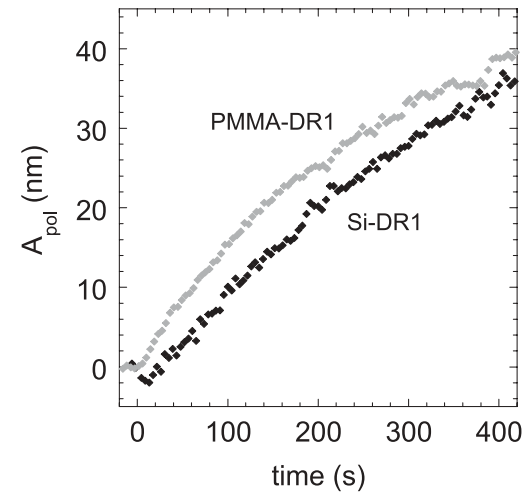

FIG. 2. Kinetics of the polarization-driven deformation process in Si-DR1 and PMMA-DR1 films. These curves are obtained from Figs. 1(h) and 1(l) by subtracting the kinetics measured when the assisting beam is off from the kinetics measured when the assisting beam is on.

configuration is kept unchanged. This observation supports the assumption of the existence of two distinct mechanisms at the origin of photoinduced mass transport which have not been considered in models proposed to describe the photomechanical response of azobenzene-containing polymers. ${ }^{8,20-32}$ The mechanism steered by the intensity pattern is revealed by the illumination with the $s$-polarized interference pattern, producing material migration directed from the bright fringes towards the dark fringes. The mechanism governed by the polarization pattern actuates directional mass transport from areas illuminated by the $E_{A} . \mathbf{x}$ component toward areas illuminated by the $E_{I} \cdot \mathbf{y}$ component. In the assisting beam illumination configuration used here, the two types of optical patterns yield mass transport in opposite directions with different kinetics and efficiencies. During the first illumination stages, the intensity-driven material migration prevails and rapidly produces a SRG of negative amplitude. It saturates quite early and gives way to the slower but more efficient polarization-driven mechanism, which reverses the mass transport direction, yielding a SRG of positive $A$ values.

If we assume that the two mechanisms are fully independent, it should be possible to single out the photodeformation kinetics $A_{\mathrm{pol}}^{\mathrm{PMMA}}(t)$ produced by the polarization pattern in the PMMA-DR1 sample. This can be done simply by subtracting the kinetics recorded without assisting beam $A_{I}^{\mathrm{PMMA}}(t)$ from the one recorded with the assisting beam $A_{A}^{\mathrm{PMMA}}(t)$. The variation of $A_{\mathrm{pol}}^{\mathrm{PMM}}=A_{A}^{\mathrm{PMMA}}-A_{I}^{\mathrm{PMMA}}$ versus time is plotted in Fig. 2 (gray symbols). It is compared with the variation of the SRG amplitude $A_{\mathrm{pol}}^{\mathrm{Si}}$ obtained in Si-DR1 (dark symbols). The two curves are very similar, with a small discrepancy partly due to the photoexpansion, which is slightly different in the two materials. The coincidence between $A_{\text {pol }}^{\text {PMMA }}$ and $A_{\text {pol }}^{\mathrm{Si}}$ leads to two conclusions. First, it is a strong indication that the polarization- and intensity-driven mechanisms are indeed fully independent from each other and that the corresponding photomechanical responses of the material simply superimpose one onto the other with their respective efficiency and direction. Second, the efficiency and kinetics of the polarization-driven photodeformation process are nearly the same in both Si-DR1 and PMMA-DR1 materials.

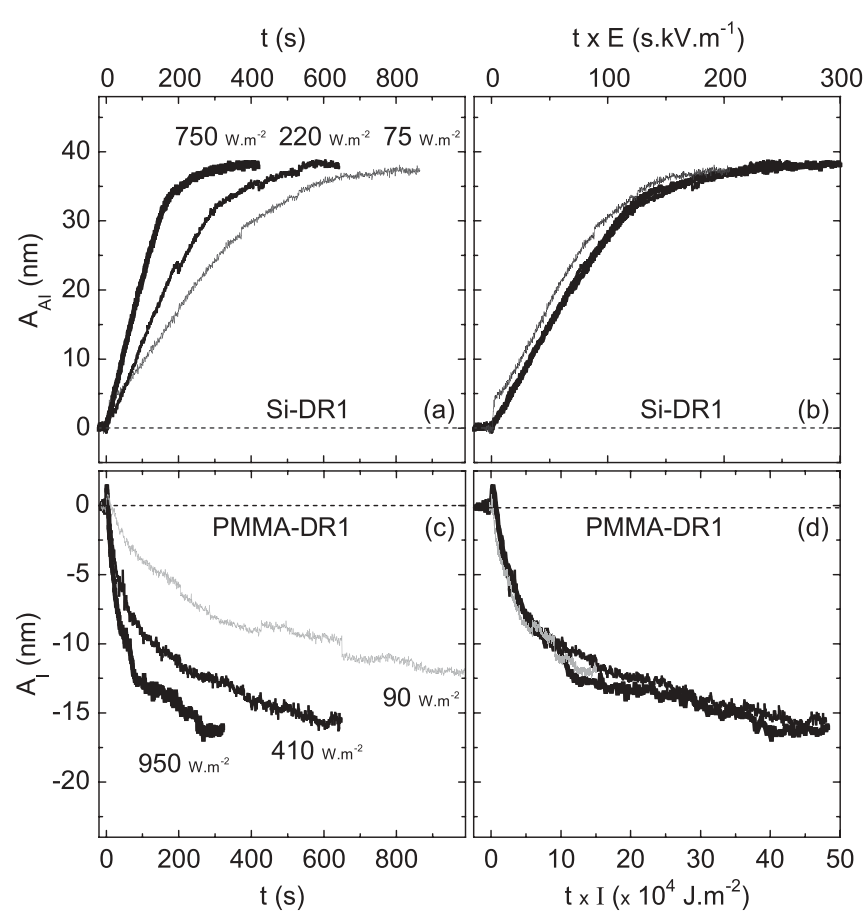

FIG. 3. (a) Kinetics of relief formation in Si-DR1 for different intensities of the assisting beam. (b) Same kinetics as (a), scaled as a function of the amplitude of the polarization pattern (i.e. the electric field modulus of the $p$-polarized assisting beam). (c) Kinetics of relief formation in PMMA-DR1 for different intensities of the $s$-polarized interference pattern. (d) Same kinetics as (c), scaled as a function of the optical power.

This contrasts with the intensity-driven mechanism whose efficiency strongly depends on the nature of the material and is completely inhibited in the sol-gel silica matrix.

Since the two processes can be independently tracked, it is interesting to study the influence of the light power on their respective efficiency. Indeed, we may expect that the efficiency of the polarization-driven process depends on the amplitude of the polarization pattern, while the efficiency of the intensity-driven process varies like the amplitude of the intensity pattern. In our configuration, the polarization-driven mass transport is triggered by the $p$-polarized assisting beam. In Si-DR1, where only this process is at play, we have measured the deformation kinetics for different values of the assisting beam power density ranging from $75 \mathrm{~W} / \mathrm{m}^{2}$ up to $750 \mathrm{~W} / \mathrm{m}^{2}$ [Fig. 3(a)]. It turns out that the deformation kinetics perfectly scales with the product $t . E_{A}$ of the exposure time by the amplitude of the assisting beam electric field [Fig. 3(b)]. Mass transport efficiency is thus governed by the amplitude of the polarization pattern, which is defined by the amplitude of the assisting beam electric field (as long as the assisting beam power density is lower than about twice the density power of the interfering beams). In contrast, the kinetics of the deformation produced by a pure intensity pattern in PMMA-DR1 for different values of the interfering beam density power $I$ [Fig. 3(c)] scales with the average density of energy $t$. I received by the material [Fig. 3(d)]. The efficiency of the mass transport process is here proportional to the amplitude of the intensity pattern. Therefore, as expected 

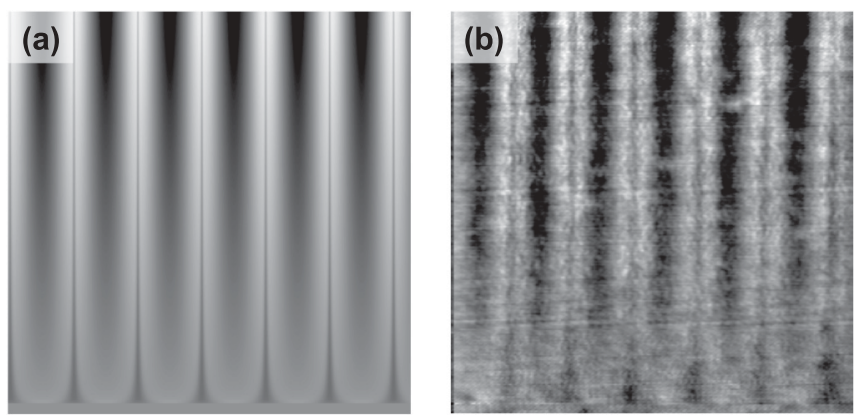

FIG. 4. (a) Calculated image of the deformation kinetics of an azobenzene-containing thin film, under the s-polarized pure intensity interference pattern. In this calculation, we consider that each photoisomerization event produces the transport of a small material volume (of the order of $\mathrm{a} \mathrm{nm}^{3}$ ) along the direction of the molecule main axis, over a distance of the order of a fraction of the molecule size. (b) Experimental image of the deformation kinetics of a PMMA-DR1 thin film under the same illumination conditions.

the two processes exhibit different dependencies on the light power density.

\section{CONCLUSION}

In the study presented here, we show that photoinduced directional mass transport in azobenzene-containing materials may be produced by two independent processes. One is driven by the light polarization pattern and the other by the light intensity pattern. We use a dedicated illumination configuration, which allows us to single out the two processes one with respect to the other. We obtain this way the kinetics of each phenomenon. It appears that the polarization-driven process almost does not depend on the nature of the matrix while the intensity-driven process is highly material dependent. In the Si-DR1 material, the intensity-driven mechanism is fully inhibited. This can be due to the 3D structure of the silica matrix network, which is different from the side-chain polymer of the PMMA-DR1 material. This can also be related to the presence of other organic moieties (here carbazole) incorporated into the silica matrix to prevent against the formation of azobenzene dimers. ${ }^{16}$

In the PMMA-DR1 material, both mechanisms are simultaneously activated and their relative efficiency and direction are controlled by setting the illumination configuration. The photoinduced deformation of the material thus results from a complex process, which may even exhibit a nonmonotonous kinetics. In the two processes, the direction and efficiency of the photoinduced mass transport are set off by different physical quantities. We come to the conclusion that the two independent mechanisms that induce optically driven mass transport in azobenzene-containing materials have different microscopic origins.

It is possible that the intensity-dependent process is directly related to the azobenzene photoisomerization. Several descriptions accounting for such a mechanism have been proposed, which could be compatible with our observation. ${ }^{24,30,31}$ In such schemes, one should expect a peculiar feature in the areas of zero light intensity, where the molecules do not photoisomerize and the material transport is thus not activated. On the basis of a simple statistical model, we have calculated the deformation of an azobenzene-containing material thin film, illuminated with an $s$-polarized pure intensity interference pattern of unitary contrast. We consider that each photoisomerization event produces the transport of a small volume of material along the direction of the molecule main axis, over a short distance. ${ }^{33}$ The elementary volume and the length of each transport step are adjustable parameters of the model. Reasonable values are typically of the order of $1 \mathrm{~nm}^{3}$ and a few tenths of a $\mathrm{nm}$ (i.e., a fraction of the molecule size). The calculated image of the surface topography during the light exposure is reproduced in Fig. 4(a). In the area corresponding to the intensity minimum of the optical pattern, we indeed observe a groove in the topography profile near the tops of the relief, which is characteristic of the absence of local photomechanical response in the dark areas. The topography image measured on a PMMA-DR1 sample [Fig. 4(b)] in the same illumination conditions exhibits the same feature. The simple statistical model of directional mass transport related to the photoisomerization rate of the chromophores provides a convincing qualitative description of the experimentally observed intensity-driven photodeformation process. This accounts for photoinduced mass transport even in the absence of an electric field component parallel to the grating vector. However, such descriptions are phenomenological and the effective microscopic origin of the mass transport as well as its material dependence are not identified.

The polarization-driven mechanism produces a surface relief pattern closely correlated with the pattern of the electromagnetic field orientation. ${ }^{12,17,34}$ Our observations are consistent with previous findings, which were interpreted in the framework of a model based on the electromagnetic forces, where photoisomerization only plays an indirect role. ${ }^{20}$ Note that, according to our results, polarization-driven material transport even occurs when the electric field component parallel to the grating vector is uniform over the entire illumination area and thus does not exhibit any gradient. This shows that one should not rely on a simplified early version of the gradient force model ${ }^{8,23}$ to describe polarization-driven mass transport.

Finally, the present demonstration of two photoinduced material transport mechanisms suggests reconsideration of the interpretation of previous experimental and theoretical results on the photomechanical response of azobenzene-containing materials. *jacques.peretti@polytechnique.edu.

${ }^{1}$ P. Rochon, E. Batalla, and A. Natansohn, Appl. Phys. Lett. 66, 136 (1995).

${ }^{2}$ D. Y. Kim, S. K. Tripathy, L. Li, and J. Kumar, Appl. Phys. Lett. 66, 1166 (1995).
${ }^{3}$ K. E. Asatryan, T. Galstian, and R. Vallée, Phys. Rev. Lett. 94, 087401 (2005).

${ }^{4}$ A. Kikuchi, Y. Harada, M. Yagi, T. Ubukata, Y. Yokoyama, and J. Abe, Chem. Comm. 46, 2262 (2010). 
${ }^{5}$ M. L. Trunov, P. M. Lytvyn, and O. M. Dyachyns'ka, Appl. Phys. Lett. 97, 031905 (2010).

${ }^{6}$ N. K. Viswanathan, S. Balasubramanian, L. Li, S. K. Tripathy, and J. Kumar, Jpn. J. Appl. Phys., Part 1 38, 5928 (1999). ${ }^{7}$ F. L. Labarthet, T. Buffeteau, and C. Sourisseau, J. Phys. Chem. B 103, 6690 (1999).

${ }^{8}$ H. Audorff, R. Walker, L. Kador, and H. W. Schmidt, J. Phys. Chem. B 113, 3379 (2009).

${ }^{9}$ D. Garrot, Y. Lassailly, K. Lahlil, J. P. Boilot, and J. Peretti, Appl. Phys. Lett. 94, 033303 (2009).

${ }^{10}$ F. Fabbri, Y. Lassailly, K. Lahlil, J. P. Boilot, and J. Peretti, Appl. Phys. Lett. 96, 081908 (2010).

${ }^{11}$ M. L. Trunov, P. M. Lytvyn, P. M. Nagy, and O. M. Dyachyns'ka, Appl. Phys. Lett. 96, 111908 (2010).

${ }^{12}$ F. Fabbri, D. Garrot, K. Lahlil, J. P. Boilot, Y. Lassailly, and J. Peretti, J. Phys. Chem. B 115, 1363 (2011).

${ }^{13} \mathrm{~A}$ combination of intensity and polarization patterns can be also achieved by interfering two laser beams, whose linear polarization is tilted by equal and opposite angles with respect to the $s$ state. However, in such a configuration, the polarization pattern can also introduce an intensity-driven mass transport. Indeed, each of the electric field components, parallel and perpendicular to the grating vector, forms an intensity interference pattern shifted one with respect to the other by half a spatial period. Thus, if we assume that the efficiency of the intensity-driven deformation depends on whether the polarization is parallel or perpendicular to the grating vector (as for instance in the inchwormlike motion scheme) ${ }^{24}$ a pure polarization interference pattern of uniform intensity distribution can produce intensity-driven mass transport. So, the polarization-driven mass transport process can hardly be isolated from the one resulting from the light intensity pattern.

${ }^{14}$ K. Yang, S. Yang, X. Wang, and J. Kumar, Appl. Phys. Lett. 84, 4517 (2004)

${ }^{15}$ C. Hubert, C. Fiorini-Debuisschert, I. Maurin, J. M. Nunzi, and P. Raimond, Adv. Mater. 14, 729 ( May 2002).

${ }^{16}$ F. Chaput, J. Biteau, K. Lahlil, J. P. Boilot, B. Darracq, Y. Levy, J. Peretti, V. I. Safarov, G. Parent, A. Fernandez-Acebes, and J. M. Lehn, Mol. Cryst. Liq. Cryst. 344, 77 (2000).
${ }^{17}$ N. Landraud, J. Peretti, F. Chaput, G. Lampel, J. P. Boilot, K. Lahlil, and V. I. Safarov, Appl. Phys. Lett. 79, 4562 (2001).

${ }^{18}$ The assisting beam configuration requires the use of two incoherent light sources to produce, on one hand, the intensity interference pattern and, on the other hand, the assisting beam. We choose two quite different wavelengths for convenience. However, two light sources of similar wavelength would not have significantly affected our results.

${ }^{19}$ P. Bertrand, L. Conin, C. Hermann, G. Lampel, J. Peretti, and V. I. Safarov, J. Appl. Phys. 83, 6834 (1998).

${ }^{20}$ K. Yang, S. Z. Yang, and J. Kumar, Phys. Rev. B 73, 165204 (2006).

${ }^{21}$ C. J. Barrett, A. L. Natansohn, and P. L. Rochon, J. Phys. Chem. 100, 8836 (1996).

${ }^{22}$ C. J. Barrett, P. L. Rochon, and A. L. Natansohn, J. Chem. Phys. 109, 1505 (1998).

${ }^{23}$ J. Kumar, L. Li, X. L. Jiang, D. Y. Kim, T. S. Lee, and S. Tripathy, Appl. Phys. Lett. 72, 2096 (1998).

${ }^{24}$ P. Lefin, C. Fiorini, and J. M. Nunzi, Pure Appl. Opt. 7, 71 (1998).

${ }^{25}$ T. G. Pedersen, P. M. Johansen, N. C. R. Holme, P. S. Ramanujam, and S. Hvilsted, Phys. Rev. Lett. 80, 89 (1998).

${ }^{26}$ S. P. Bian, J. M. Williams, D. Y. Kim, L. A. Li, S. Balasubramanian, J. Kumar, and S. Tripathy, J. Appl. Phys. 86, 4498 (1999).

${ }^{27}$ D. Bublitz, B. Fleck, and L. Wenke, Appl. Phys. B 72, 931 (2001).

${ }^{28}$ T. M. Geue, M. G. Saphiannikova, O. Henneberg, U. Pietsch, P. L. Rochon, and A. L. Natansohn, Phys. Rev. E 65, 052801 (2002).

${ }^{29}$ D. Barada, M. Itoh, and T. Yatagai, J. Appl. Phys. 96, 4204 (2004).

${ }^{30}$ B. Bellini, J. Ackermann, H. Klein, C. Grave, P. Dumas, and V. Safarov, J. Phys.: Condens. Matter 18, S1817 (2006).

${ }^{31}$ M. L. Juan, J. Plain, R. Bachelot, P. Royer, S. K. Gray, and G. P. Wiederrecht, Appl. Phys. Lett. 93, 153304 (2008).

${ }^{32}$ V. Toshchevikov, M. Saphiannikova, and G. Heinrich, J. Phys. Chem. B 113, 5032 (2009).

${ }^{33}$ See Supporting Information in F. Fabbri et al., J. Phys. Chem. B 115, 1363 (2011).

${ }^{34}$ T. Grosjean and D. Courjon, Opt. Express 14, 2203 (2006). 